definition remark Example [][]erfc 


\title{
Two-phase Stefan problem with nonlinear thermal coefficients and a convective boundary condition
}

\author{
Adriana Briozzo ${ }^{1}$ and Maria Natale ${ }^{1}$ \\ ${ }^{1}$ Universidad Austral Buenos Aires - Sede Rosario
}

May 5, 2020

\begin{abstract}
We consider a non-linear two-phase unidimensional Stefan problem, which consists on a solidification process, for a semi-infinite material $x>0$, with phase change temperature $T_{1}$, an initial temperature $T_{2}>T_{1}$ and a convective boundary condition imposed at the fixed face $x=0$ characterized by a heat transfer coefficient $h>0$. We assume that the volumetric heat capacity and the thermal conductivity are particular nonlinear functions of the temperature in both solid and liquid phases and they verify a Storm-type relation. A certain inequality on the coefficient $h$ is established in order to get an instantaneous phase change process. We determine sufficient conditions on the parameters of the problem in order to prove the existence and uniqueness of a parametric explicit solution for the Stefan problem.
\end{abstract}

\section{Hosted file}

BrNa2020.pdf available at https://authorea.com/users/300897/articles/430646-two-phasestefan-problem-with-nonlinear-thermal-coefficients-and-a-convective-boundary-condition 